Military Technical College

Kobry El-Kobbah, Cairo, Egypt.

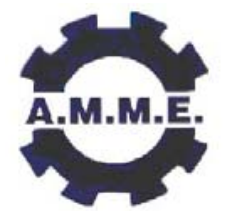

$13^{\text {th }}$ International Conference on Applied Mechanics and Mechanical Engineering.

\title{
MODELING CRYSTALLINE STRUCTURE FOR METALS USING A THREE DIMENSIONAL SIMULATION CODE: PART I
}

ELSHARKAWY ${ }^{*}$ S.G. and SHEHADEH ${ }^{* *}$ M.F.

\begin{abstract}
The field of crystalline structure research is considered as an important field in material technology for making a good decision on mechanical engineering design. This paper presents a code for simulating a crystal structure of crystalline materials. This code enables to avoid implications due to difficulty of analytical methods. The 3D of a crystalline structure for a body-centered cube (BCC) Fe-metal lattice is simulated. The physical inputs into the model are number of atoms, atomic volume, and vacancyformation energy. This code simulates the deviation of atoms orientation as well as the estimation of relative embedded energy at different temperature values. The new atom poistions have been traced, also, the transition phase of $\mathrm{Fe}$ crystalline structure is pointed out.
\end{abstract}

\section{KEY WORDS}

Modeling, Crystalline material, Crystal structure, Lattice, Energy, Point defects, Simulation, Iron, BCC, FCC.

* Ass. Prof., Dpt. of Basic Science Studies, AASTMT, Alexandria, Egypt.

** Ass. Prof, Dpt. of Mechanical and Marine Engineering, AASTMT, Alexandria, Egypt. 


\section{INTRODUCTION}

For all engineering fields, materials are playing an important role to develop engineering science. In the last decade, due to computer development, technologies of engineering materials have exclusively been developed by computer simulation methods [1,2]. The usage of computer simulation techniques based on modeling approaches offer the opportunity to simulate the properties of real materials, making prediction of new phenomena and explaining experimental observations. Consequently, constructing simulation techniques for a three dimensional lattice crystal has been a subject of significant interest in all fields of technology. There are numerous challenges and several major obstacles to overcome before such simulations are established.

The development of crystalline structure in three dimensions requires better understanding of the crystallization kinetics of different materials. A lot of the existing simulation methods are based on two-dimensional crystallization models. Zhaohui Fan et al [3] have developed a method to simulate the crystallization of phase change recording material in three dimensions. Their method was used to simulate the recording layer thickness effect on the crystallization. Monte Carlo simulations were carried out by Xu J. and Selinger R. L. B. [4] to investigate the effect of molecular shape on liquid crystal order. Zhao P. et al [5] have simulated thermally driven crystal growth in three dimensional space. They formulated the model using finite element method, and achieved it to work directly with primitive variables. They also solved the energy equation in a fixed volume mesh which tracks the motion of the solid-liquid interface. A simulation program of a three dimensional anisotropic etching profile was developed by Koide et al [6] to design the fabrication processes of microstructure made of single crystal silicon. Chen D. [7] has presented a computer model technique to provide details of structure, energy and elastic properties of nano-crystals. Their simulation was agreed with experimental results which indicated that the computer simulation method is an available technique for the study of structures and properties of crystalline materials.

It is well known that, the properties of crystalline materials are dependent on their crystal structure, which is termed anisotropy [8,9]. Accordingly, understanding these structures and physical properties allow us to predict how that material will behave under certain conditions [10,11-13]. For example, Vinogradov and Telitchev [14] have studied the phenomenon of hydrogen embrittlement for body-centered cube (BCC) iron using a computer simulation. They noted that, both distortions of a BCC iron lattice structure and loss of strength occur as a result of the presence of atomic hydrogen molecules near the crack tip.

Materials have many different crystal structures which consist of atoms arranged in a pattern that are repeated periodically in a three-dimensional geometric lattice [8]. Consequently, the structure of such materials may be derived from a small unit of atoms 'unit cell'. Therefore, the properties of the whole material can be completely described by considering just one unit cell containing a small number of atoms. Few, if any, crystals are perfect in that all unit cells consist of the ideal arrangement of atoms or molecules and all cells line up in a three dimensional space with no distortion. Some cells may have one or more atoms less or more than the ideal unit cell. However, it is almost impossible to obtain a perfectly pure material. The imperfections of crystals are called crystal defects. Many researchers have studied such structures analytically and numerically $[10,12,13-17]$. 
Pure iron is of great importance to engineering applications (e.g. Structures and automobiles), the material studied in this paper, and perhaps it's most obvious and common application is its combination with carbon to produce steel. It could be argued that steel is one of the most widely used metals in the world. Pure iron can exist in two different atomic structures, body-centered cube (BCC) and face-centered cube (FCC) [8]. This paper presents a computer technique to simulate and analyze the crystalline materials of pure iron "BCC" in three dimensions.

\section{POINT DEFECTS IN CRYSTALLINE MATERIALS}

Point defects "missing atom" strongly affect almost all properties of crystalline materials, which include self-interstitial atoms, interstitial impurity atoms and vacancies. In general, the diffusion (mass transport by atomic motion) can only occur because of vacancies. Embedded Atom Method (EAM), discussed in the next section, have been used by many papers to investigate the interaction between point defects (both vacancies and anti-site atoms) $[15,17,19]$.

During the defects' interaction, the plastic deformation usually occurs due to the motion of dislocations, point defects and twins [8,20]. Also, the effects of point defects to generate new properties of submicro-crystalline and nano-structure in metals have been studied recently [19-25]. Kido and Tani [22] have studied analytically the point defects in $\beta-F e S i$, where transition metal silicides are considerable. They noted that the formation energies of the point defects depend largely on the atomic chemical potentials of $\mathrm{Fe}$ and $\mathrm{Si}$.

Vacancies are known as an empty space that would be occupied by an atom. This case commonly happens, especially at high temperatures, atoms are frequently and randomly changing their positions leaving behind empty lattice sites $[19,20]$. The concentration of vacancies $(\mathrm{N} v)$ in a crystal has an Arrhenius dependence on temperature and expressed by:

$$
N_{v}=N e^{\frac{Q_{v}}{K T}}
$$

Where $N$ is the concentration of lattice sites, $Q_{V}$ is the formation energy of the vacancy in eV/atom, $K$ is the Boltzmann's constant and $T$ is the absolute temperature in Kelvin. Increasing the temperature of a solid causes the vacancy concentration to increase exponentially. Consequently, increasing vacancy concentration allows high temperature diffusion to take place in materials (i.e. control the impurity concentration in semiconductors during manufacturing) [26].

\section{INTERATOMIC POTENTIAL ENERGY MODELL}

As mentioned before, many of the crystalline material properties originate from atomic arrangements. Therefore, for an elaborate control of material properties, it is necessary to investigate and understand the material's behavior from an atomic level. The embedded atom method (EAM) proposed by many researchers $[9,15,17]$ is a semi empirical method for describing the energy between two atoms. Narayan et al. [17] have used the EAM to point the physical properties of pure metal crystals. They 
indicted that the method is easy to implement. At the same time, it accurately captures the physical "thermal and elastic" behavior of BCC and FCC crystal lattice. Predicting correct values of Young modulus, Poisson's ratio, lattice constants at various temperatures are examples for the variables predicted by using such a method. Nogueira and Schön [15] have studied diffusion by EAM and molecular static's atomistic simulations in iron-aluminizes (Fe-Al) compounds. Also, Chamati et al. [27] have studied the self-diffusion of single atom on the BCC Fe $\left(\begin{array}{lll}1 & 0 & 0\end{array}\right)$ surface by using a developed EAM potential and molecular dynamics simulation in temperature range of 600-950 K.

The EAM represents the total energy of a monoatomic system as $[15,27]$ :

$$
E_{E A M}=\frac{1}{2} \sum_{i j} V\left(r_{i j}\right)+\sum_{i} F\left(\overline{\rho_{i}}\right)
$$

where $V\left(r_{i j}\right)$ is the pair interaction energy between atoms $i$ and $j$ separated by a distance $r_{i j}$ and $F$ is the embedding energy of an atom $i$ as a function of the host electron density $\rho_{i}$. The latter is given by

$$
\bar{\rho}=\sum_{i \neq j} \rho\left(r_{i j}\right)
$$

where $\rho(r)$ is the electron density function assigned to an atom.

\section{SIMULATION METHODOLOGIES}

A code is written by MATLAB program and the work only focuses on simulating the vacancies in crystal structure of BCC. The code is generated to simulate the crystal structure in three dimensional modes. With the help of this code, the influence on the effective geometrical sizes of structural elements of a crystal and the thermodynamic parameters of crystallization can be simulated.

The behavior of different atoms is based on the principle of the order in the unit cell and their neighbors. So, each atom has an exact position in the three dimensions $\left(x_{1}, y_{1}, z_{1}\right)$ relative to the others in the unit cell. An arbitrary cell point (origin) is chosen and three mutually perpendicular edges of the unit cell were taken as coordinate axes. For a lattice constant (a) defined as the length of the cell edge, a simple cubic lattice with a vector $\mathrm{R}$ is written as follows:

$$
R=n_{1} a \vec{x}+n_{2} a \vec{y}+n_{3} a \vec{z}
$$

The code simulates a shape of a simple lattice where the conventional cubic cell is a primitive cell. The probabilities of motion and transition of atoms from their initial positions are calculated on the bases of the thermal activation energy equation. The length of the distance moved by an atom is calculated through the relation given by:

$$
\text { Dis } \tan c e=\text { Time } \times \sqrt{\frac{3 K T}{M}}
$$


where $K$ is the Boltzmann's constant, $T$ is the absolute temperature in Kelvin, and $M$ is an atomic weight for iron.

It presumes that, atoms perform thermal motion and could accept enough energy to overcome the forces among its neighboring atoms to reach new position of coordinates $\left(\mathrm{X}_{2}, \mathrm{Y}_{2}, \mathrm{Z}_{2}\right)$. The flowchart of the program developed is shown in Fig.1.



Fig.1. The code flowchart

\section{RESULTS AND DISCUSSION OF SIMULATION}

A computer code has been constructed using MATLAB to simulate the actual BCC unit cell in an iron crystal. The constants of the unit cell are the inter-atomic spacing and the 
atomic diameter which have the values of $2.87 \times 10^{-10} \mathrm{~m}$ and $0.124 \times 10^{-9} \mathrm{~m}$ respectively. The coordinates of each atom have been registered with respect to the origin. Avarge of five run trials have been executed by the code to present the reliability of simulation and decrease the error percentage, Fig. (2-6).

An arrangement of BCC crystal atoms is described in Fig.2, showing that, a typical BCC crystal type in a well arranged matrix was generated. The spacing among atoms were taken exactly as that of the real atomic spacing measured in reality. Each atom has been marked with a reference number as well as lattice coordinates to be traced with. Also, atoms can be simulated in two dimensions as shown in Fig.3.

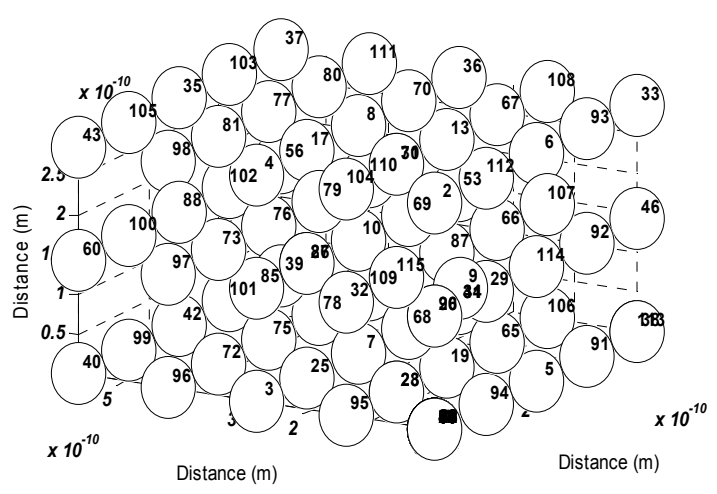

Fig.2. The output simulation shaping for an array of atoms for BCC iron crystal

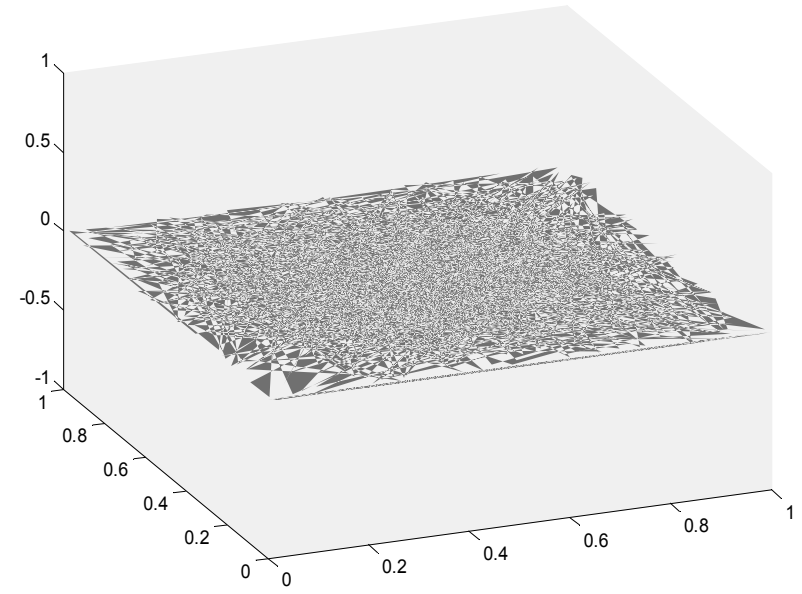

Fig.3. Representation of a plane surface for the simulated atoms

The result of a three dimensional simulation for a typical BCC iron unit cell is shown in Fig.4. The intermolecular spacing to atomic size ratio has been taken into consideration in the representation of all figures in this paper. So, to demonstrate clearly the position of each atom and the difference in initial and final position of the central atom before and after heating, it's been decided not to apply the real ratio in representing the figures.

The new atomic coordinates of the central atom "atom number 9" has been simulated, Fig.5, to show the effect of applying heat on this atom. This result wouldn't be obtained in either motion of one or two dimensional simulations. Through this code, the tracing of any atom as well as studying the influence of applying any external effect can be executed. This lead us to believe that, this code is an alternative way to the laboratory work.

The normalized distance for BCC central atom against the normalized temperature is plotted in Fig.6. The examined temperatures have been chosen to cover the range of $500^{\circ} \mathrm{C}$ to $950^{\circ} \mathrm{C}$, with $50^{\circ} \mathrm{C}$ increment. Upper temperature of the range has been taken due to the theoretical critical transitional limit between BCC and FCC modes, where FCC inflection mode for iron crystals is completed around $950^{\circ} \mathrm{C}$. Three regions can be 


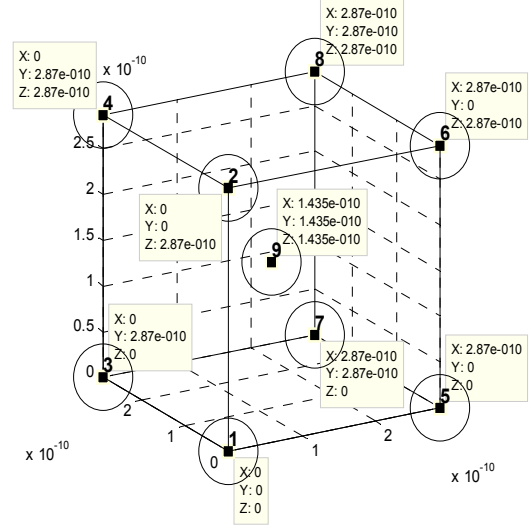

Fig. 4. Output graphical shape simulation of BCC iron unit cell in three dimensions.

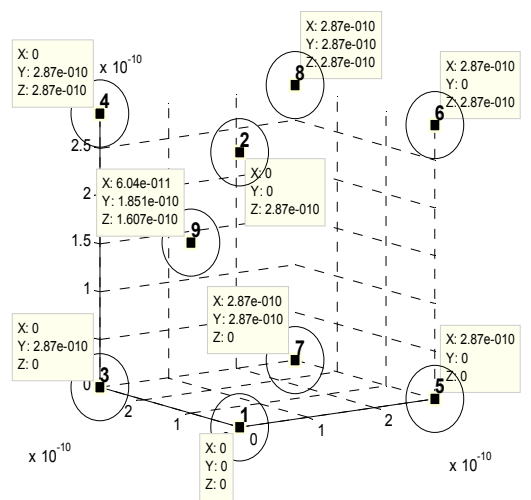

Fig. 5. Output simulation result after applying heat concept to atom 9.

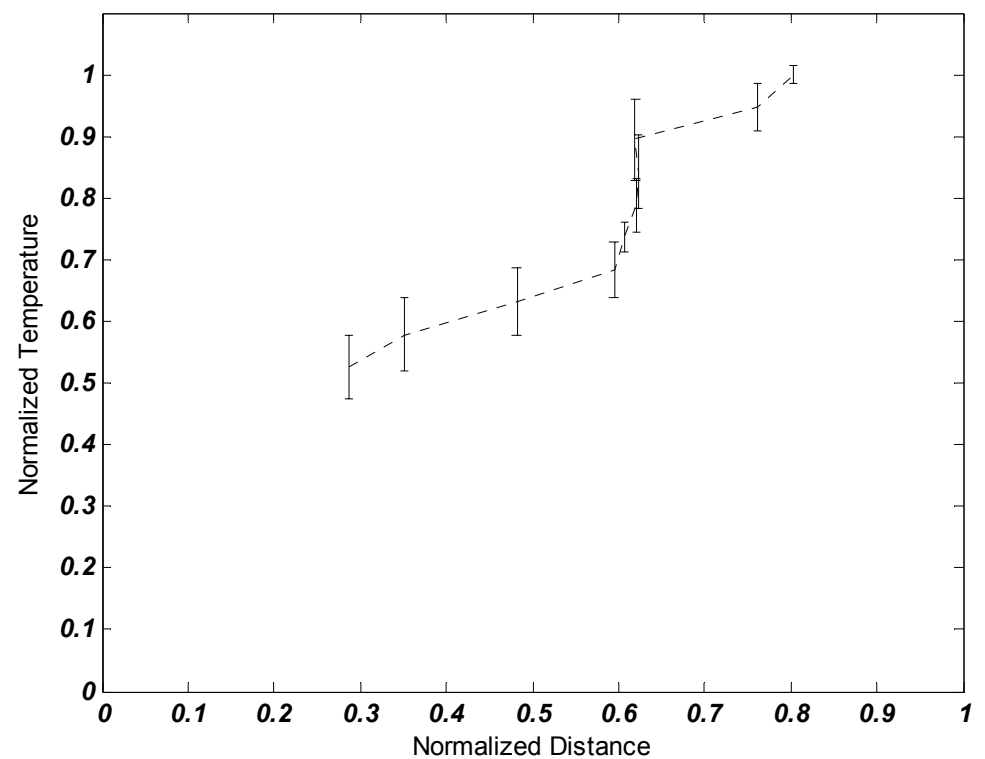

Fig. 6. Atomic distance as a function of temperature for central atom in BCC.

identified on Fig.6, region I " $500^{\circ} \mathrm{C}-650^{\circ} \mathrm{C}$ ", region II " $650^{\circ} \mathrm{C}-850^{\circ} \mathrm{C}$ " and region III "above $850^{\circ} \mathrm{C}$ ". Firstly, region I, the distance moved by the central atom is represented by the first three points on the graph. The atomic distance was increased due to the rise in temperature, as a result of the central atom became closer to the edge of its unit cell. Secondly, region II, the next five points on the graph, the distance moved by the atom is increased in small increments compared to those in region I. This is interpreted as the starting range for the unit cell to become transformed from BCC to FCC phase structure. Finally, region III, shows the same behavior as in region I. This is explained as the completion of change into FCC phase structure by the central atom. 


\section{CONCLUSIONS}

A three dimensional simulation of a typical BCC unit cell for an iron crystal has been demonstrated by a new code. It was shown from the simulation results that the central atom was affected by heat and started to move to new randm positions. The distances moved by the atom were calculated and plotted against the normalized temperature. These new atom positions were identified; and the change from the BCC phase structure to FCC phase structure was pointed out "region III". This simulation offers the chance to test different materials from nano-scale point of view and achieve different "simulation-laboratory" analogues. Finally, the effort done through this paper is considered as the beginning of series investigations for different crystalline materials (e.g. properties, parameters, conditions, .... etc.).

\section{REFERENCES}

[1] Vitosa L., Korzhavyia P.A. and Johanssona B. Modeling of Alloy Steel. Material Today J, October, pp. 14-23, 2002.

[2] Vashishta P., Kalia R. K., Nakano A., Multimillion atom simulations of dynamics of wing cracks and nanoscale damage in glass, and hypervelocity impact damage in ceramics. Computer Physics Communications, 177, pp. 202-205, 2007.

[3] Fan Z. and Laughlin D. E., Three Dimensional Crystallization Simulation and Recording Layer Thickness Effect in Phase Change Optical Recording, .J. .Appl. .Phys., 42 (1), pp. 800-803, 2003.

[4] Xu J. and Selinger R. L. B., Monte Carlo Simulation of liquid-crystal Alignment and of Chiral Symmetry-Breaking, J. Chem. Physics, 115, 4333, 2001.

[5] Zhao P., Heinrich J. C. and Poirier D. R., Fixed Mesh Front-Tracking Methodology for Finite Element Simulation, International Journal for numerical methods in engineering, 71 (1), pp. 25-46.

[6] Koide A. and Tanaka S., Simulation of three-dimensional etch profile of silicon during orientation dependent anisotropic etching. MicroElectroMechanical systems, 1997 MEMS apos; 97, proceedings IEEE; $10^{\text {th }}$ annual international workshop, 26-30 (Jan), pp. 418-423, 1997.

[7] Chen D., Computer model simulation study of nanocrystalline iron. Material Science and Engineering A., 190, pp. 193-198, 1995.

[8] Callister W. D., Material Science and Engineering an Introduction. John \& Wiley Sons Inc., $7^{\text {th }}$ ed, 2007.

[9] Wang D., Zhang J. and Xu K., Anisotropy analysis of the surface energy of hcp (c/a < 1.633) metals. Surface Science J., pp. 2990-2996, 2006.

[10] Zhang J., Li H., Xu K., Reconstructed (1 110$)$ surfaces of FCC transition metals. Journal of Physics and Chemistry of Solids, 67, pp. 1623-1628, 2006.

[11] Li X.Y. W. D.Y., Mechanical, electrochemical and tribological properties of nanocrystalline surface of 304-stainless steel. Wear Journal, 255, pp. 836-845, 2003.

[12] McDowell D. L., Simulation-based strategies for microstructure-sensitive fatigue modeling. Material Science and Engineering A, 468-470, pp. 4-14, 2007.

[13] Kim H. S. and Estrin Y., Strength and strain hardening of nanocrystalline materials. Material Science and Engineering A, 2007.

[14] Vinogradov O. and Telitchev I. Y., Numerical tensile tests of BCC iron crystal with various amounts of hydrogen near the crack tip. Computational Material Science, 36, pp.272-280, 2006. 
[15] Nogueira R. N., and Schön G. C., Embedded atom method study of the interaction between point defects in iron aluminides: Triple defects. Intermetallics J., 13, pp. 1245-1254, 2005.

[16] CHEPULSKII R. V., COMPARISON OF TWO ANALYTICAL APPROXIMATIONS FOR CALCULATION OF THE SHORT-RANGE ORDER IN DISORDERED ALLOYS. J. Phys. Chem. Solids, 59(9), pp. 1473-1476, 1998.

[17] Narayan K., Behdinan K. and Fawaz Z. An engineering-oriented embedded-atommethod potential fitting procedure for pure fcc and bcc metals. Journal of Materials Processing Technology, 182, pp. 387-397, 2007.

[18] Merwe J.H., Interplay between misfit strain relief and Stranski-Krastanov growth in fcc (111)/bcc (110) ultrathin film epitaxy Part 1. Analytical approach. Surface Science, 449, pp. 151-166, 2000.

[19] Zhang J.M., Shu Y. and Xu K. W., Multilayer relaxation of fcc metals (001) surface: A modified embedded atom method study. Solid State Communications, 137, pp. 441-445, 2006

[20] Gao H. and Ji B., Modeling fracture in nanomaterials via a virtual internal bond method. Engineering Fracture Mechanics, 70, pp. 1777-1791, 2003.

[21] Muižnieks A., Madžulis I., Dadzis K. and Mühlbauer A., Simplified Model for Monte Carlo Simulation of Point Defect Dynamics during Industrial Silicon Crystal Growth. International Scientific Colloquium "Modeling for Electromagnetic Processing", Hanover, pp. 311-316, 2003.

[22] Morris M.A. and Morris D.G., Quenching and Ageing Effects of Defects and Their Structures in FeAl Alloys, and the Influence of Hardening and Softening. Acta Metallurgica Inc., 38(3), pp. 509-516, 1998,.

[23] Tani J. and Kido H., First-principle study of native point defects in $\beta$-FeSi2. Journal of Alloys and Compounds, 2003, 352, pp. 153-157.

[24] SO R., and KURZYDLOWSKI K. J., Atomic modeling of point defects in B2-RuAl. Materials Science, 23 (2) , 2005.

[25] Ren X. and Otsuka K., Universal Symmetry Property of Point Defects in Crystals. PHYSICAL REVIEW LETTERS, 85 (5), pp. 1016-1019, 2000.

[26] Kim E. H., Shin Y. H. and Lee B. J., A modified embedded-atom method interatomic potential for Germanium. Computer Coupling of Phase Diagrams and Thermochemistry, 2007.

[27] Chamati H., Papanicolaou N.I., Mishin Y.and Papaconstantopoulos D.A., Embedded-atom potential for $\mathrm{Fe}$ and its application to self-diffusion on Fe (100). Surface Science, 600, pp.1793-1803, 2006. 